\title{
Trombosis primaria de la descendente anterior en un paciente con síndrome de anticuerpos antifosfolípidos
}

Jorge Acuña-Valerio ${ }^{1}$, Marco Antonio Peña-Duque ${ }^{2}$ y Álvaro Contreras-Villaseñor ${ }^{2}$

${ }^{1}$ Programa de Maestría y Doctorado en Ciencias Médicas, Odontológicas y de la Salud, Universidad Nacional Autónoma de México, con sede en el Instituto Nacional de Cardiología Ignacio Chávez; ${ }^{2}$ Departamento de Hemodinámica, Instituto Nacional de Cardiología Ignacio Chávez. Ciudad de México, México

\section{Resumen}

El síndrome de anticuerpos antifosfolípidos es una situación clínica y bioquímica heterogénea. Presentamos el caso de un varón joven con antecedente de tromboembolia venosa que se presentó en esta ocasión por dolor precordial, con elevación del ST en el electrocardiograma. Fue llevado a sala de angiografía para realizar angioplastia primaria y se observó una oclusión total ostial de la descendente anterior. Se realizó aspiración manual del trombo. No se realizó angioplastia con balón ni stent. En la angiografía de control a las 48 horas se observó ausencia de trombo y de placas aterosclerosas, lo cual se corroboró mediante ultrasonido intracoronario.

PALABRAS CLAVE: Síndrome antifosfolipídico. Anticuerpos antifosfolípidos. Trombosis coronaria. Infarto agudo del miocardio. Fenómeno de no reflujo.

\begin{abstract}
Antiphospholipid antibody syndrome is a heterogeneous clinical and biochemical entity. We present the case of a young male with history of venous thromboembolism. This time he presents because of chest ischemic pain associated with ST segment elevation. He was taken to the cath lab to perform a primary percutaneous coronary intervention and a total occlusion of the left anterior descending artery was noted. Successful thrombus aspiration was performed. No stent was deployed. He was taken to the cath lab for a second look angiography and no atherosclerotic lesions were observed, which was confirmed by intravascular ultrasound.
\end{abstract}

KEY WORDS: Antiphospholipid antibody syndrome. Antiphospholipid antibodies. Coronary thrombosis. Acute myocardial infarction. Non-reflow phenomenon.

Fecha de recepción: 09-06-2016

Fecha de aceptación: 10-06-2016

DOI:10.24875/GMM.17002572
Gac Med Mex. 2017;153:526-530

Contents available at PubMed www.gacetamedicademexico.com 


\section{Introducción}

El síndrome de anticuerpos antifosfolípidos (SAF), o síndrome de Hughes, es una situación heterogénea que se refiere a: 1) presencia de anticuerpos contra los fosfolípidos o las proteínas fijadoras de fosfolípidos, los cuales se conocen como anticuerpos antifosfolípidos (AFL), de entre los que están mejor caracterizados el anticoagulante lúpico, la anticardiolipina y la anti-ß32-glucoproteína; 2) cuando menos una de las manifestaciones clínicas, que incluyen trombosis arteriales o venosas, pérdidas fetales y trombocitopenia $^{1,2}$. Los criterios para clasificar a los pacientes fueron publicados en 1999 y revisados en $2006^{3}$.

Se puede presentar asociado a otras enfermedades autoinmunes (principalmente lupus eritematoso sistémico), conociéndose como SAF secundario, pero hasta en el $50 \%$ de los casos se manifiesta como un síndrome clínico único, clasificándose como SAF primario. Otra forma clínica menos común es el SAF catastrófico ${ }^{4}$.

Las manifestaciones cardiovasculares más frecuentes del SAF son las trombosis arteriales o venosas. Sin embargo, también se han asociado con el síndrome aterosclerosis acelerada, endocarditis verrugosa, alteraciones valvulares cardiacas, trombosis intracardiaca con o sin cardioembolia, trombosis de stent, pericarditis con o sin afección del miocardio, degeneración prematura de puentes venosos, hipertensión pulmonar, hipertensión arterial secundaria a estenosis renal y enfermedad arterial periférica ${ }^{5}$. Las manifestaciones cardiovasculares varían según se trate de SAF primario o asociado a otras enfermedades autoinmunes ${ }^{6}$.

\section{Presentación del caso}

Varón de 27 años de edad, quien había presentado trombosis venosa profunda iliofemoral izquierda 4 años antes (no provocada), con subsecuente tromboembolia pulmonar. Este cuadro no se asoció con ningún factor de riesgo clínico para tromboembolia y la detección de AFL fue positiva. Se trató con heparina convencional y posteriormente con cumarínico, y se colocó un filtro de vena cava inferior. Se mantuvo asintomático y en tratamiento anticoagulante.

En esta ocasión presentó dolor precordial opresivo de aparición abrupta al caminar, por lo que acudió al hospital. Se observó elevación del segmento ST en las derivaciones de pared anterior del electrocardiograma.
Se administraron $300 \mathrm{mg}$ de ácido acetilsalicílico y $300 \mathrm{mg}$ de clopidogrel, y se refirió a un centro con capacidad para realizar angioplastia primaria. Llegó a nuestro hospital con un tiempo de isquemia de 3 horas. Se observó taquicárdico (110 lpm), taquipneico (26 vpm) y diaforético, sin signos de falla cardiaca aguda ni de otra alteración cardiopulmonar significativa. Un nuevo electrocardiograma demostró elevación del ST en V1-V5, DI y aVL. Se administraron morfina, oxígeno, otros $300 \mathrm{mg}$ de clopidogrel y $4000 \mathrm{UI}$ de heparina intravenosa (su último INR [international normalized ratio] era de 1.5, una semana antes), y fue llevado a la sala de hemodinámica. Se seleccionó el abordaje radial.

En la coronariografía se observó una oclusión total ostial de la descendente anterior (trombo grado 5) (Fig. $1 \mathrm{~A}$ y $1 \mathrm{~B}$ ). No se detectaron otras alteraciones coronarias. Se avanzó una guía Runthrough floppy (Teru$\mathrm{mo}^{\circledR}$ ), la cual cruzó la obstrucción sin dificultad, y se avanzó un sistema de aspiración manual Diver (Invatec $\left.^{\circledR}\right)$, con el que se extrajeron varios fragmentos de material trombótico y se restauró la permeabilidad del vaso (Fig. 2 A y 2 B). El tiempo puerta-balón fue de 74 minutos. Se repitió la aspiración de trombo dos veces más y se administró una dosis doble de tirofibán intracoronario. Se observó fenómeno de no reflujo leve y se utilizó adenosina, con lo cual mejoró. En las angiografías subsecuentes en diversas proyecciones no se logró identificar ninguna placa aterosclerosa, el trombo residual era mínimo y el flujo casi normal (TIMI 3 MBG 2), por lo que se decidió no implantar un stent. Se continuaron las infusiones periféricas de heparina y tirofibán. Se observaron criterios de resolución (desaparición total del dolor y normalización de la elevación del segmento ST). Ocho horas después presentó melena, por lo que se realizó una endoscopia de tubo digestivo alto, en la cual se observó gastropatía difusa asociada a estrés. El ecocardiograma reveló una fracción de eyección del ventrículo izquierdo (FEVI) del $34 \%$, hipocinesia moderada anterior y apical, disfunción diastólica leve, no se identificaron alteraciones valvulares o pericárdicas, y la presión pulmonar se estimó en $42 \mathrm{mmHg}$. La radiografía mostró signos de congestión pulmonar. El valor máximo de troponina I fue de $>150 \mathrm{ng} / \mathrm{ml}$. Se indicaron vasodilatadores y diuréticos, y evolucionó satisfactoriamente sin necesidad del uso de inotrópicos.

A las 48 horas fue llevado de nuevo a la sala de angiografía, en donde se realizó coronariografía izquierda. La imagen de trombo residual desapareció. No se observaron lesiones coronarias. Se avanzó una 

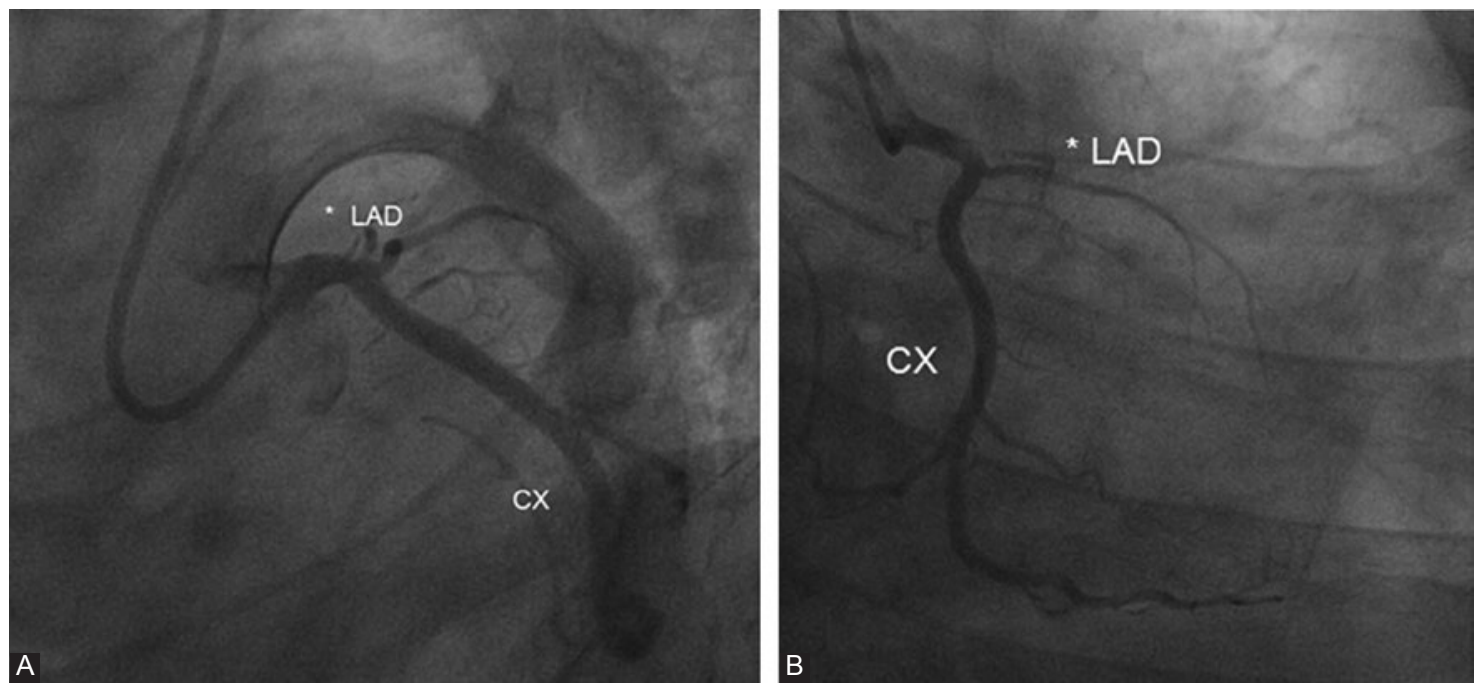

Figura 1 A y 1 B. Oclusión total de la descendente anterior desde su ostium. Circunfleja sin lesiones. No se muestra la coronaria derecha, que tampoco tenía lesiones. CX: arteria circunfleja; LAD: arteria descendente anterior.
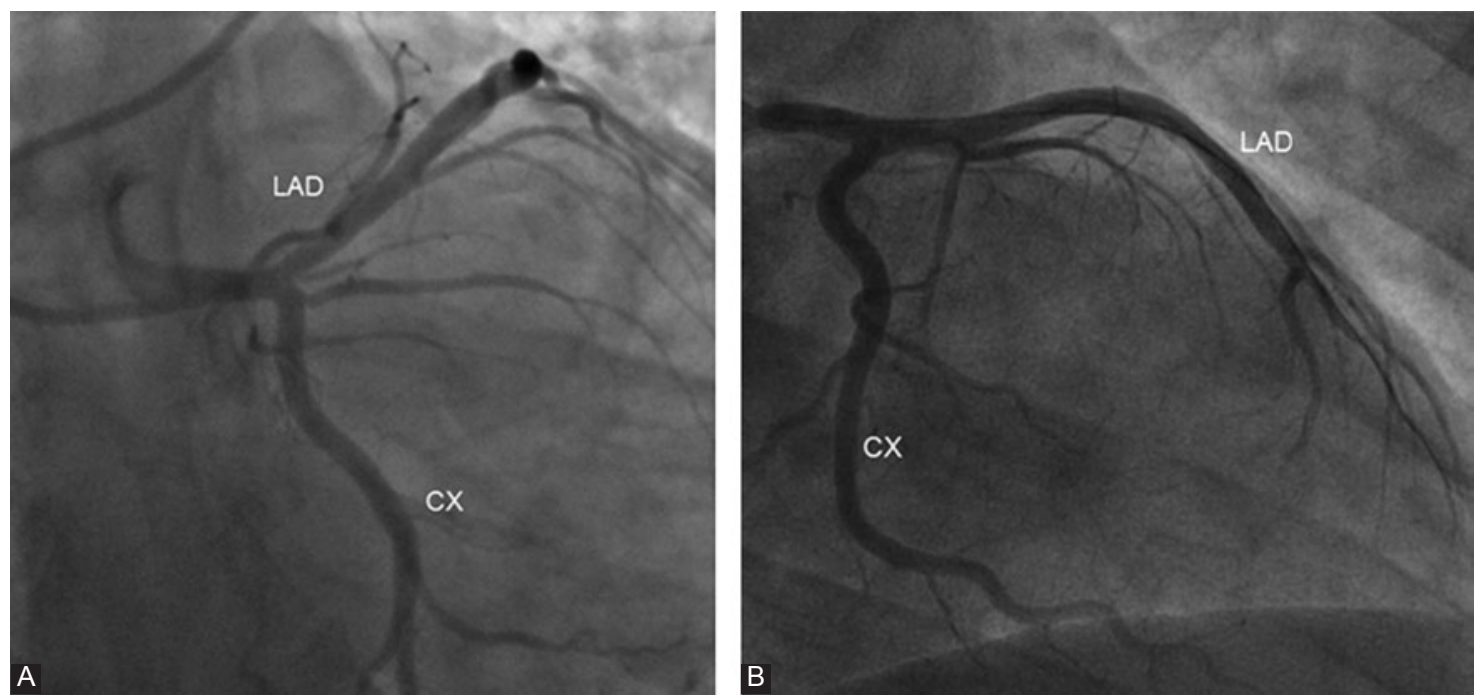

Figura 2 A y 2 B. Después de la aspiración manual, la descendente anterior recuperó el flujo y no se observaron estrechamientos sugerentes de aterosclerosis. Hay un trombo laminar pequeño en el ostium (sin fenómenos de embolia en ramas distales). En la arteriografía practicada 2 días después el trombo había desaparecido por completo. CX: arteria circunfleja; LAD: arteria descendente anterior.

sonda de ultrasonido intracoronario $\left(V_{0}\right.$ Veano $\left.^{\circledR}\right)$ y no se encontraron placas ateroscleróticas en la corrida ultrasonográfica en la descendente anterior ni en el tronco coronario izquierdo. Como se había detectado disminución del pulso femoral derecho desde el ingreso, se realizó angiografía iliofemoral derecha, la cual documentó oclusión de $<5 \mathrm{~cm}$ de la iliaca externa y femoral común, con recanalización subsecuente y circulación colateral adecuada. Dado que estaba asintomático y la obstrucción no era aguda, se decidió no realizar ninguna intervención en ese momento.

No volvió a presentar dolor precordial, cambios electrocardiográficos sugerentes de isquemia ni nuevas elevaciones enzimáticas. Tampoco presentó otra hemorragia anormal. Al interrogatorio dirigido no se encontró antecedente familiar de enfermedad coronaria prematura ni otro factor de riesgo cardiovascular, así como tampoco síntomas sugerentes de enfermedad autoinmune. El departamento de reumatología lo valoró y no encontró alteraciones sugerentes de lupus eritematoso sistémico ni de ninguna otra enfermedad autoinmune. Los anticuerpos antinucleares y el factor reumatoide fueron negativos, la lgM anticardiolipina fue de $8.4 \mathrm{MPL} / \mathrm{ml}$ (normal $<7 \mathrm{MPL} / \mathrm{ml}$ ), la IgG anticardiolipina fue de $>120 \mathrm{GPL} / \mathrm{ml}$ (normal

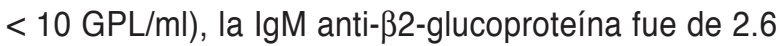
$\mathrm{U} / \mathrm{ml}$ (normal $<8 \mathrm{U} / \mathrm{ml}$ ), la IgG anti-ß2-glucoproteína 
fue de $>100 \mathrm{U} / \mathrm{ml}$ (normal $<8 \mathrm{U} / \mathrm{ml}$ ), y el anticoagulante lúpico fue positivo. Basándose en la historia clínica y en este patrón bioquímico, y en el antecedente de una pesquisa para AFL que había sido positiva, se clasificó como SAF primario. Antes del alta hospitalaria se realizó un nuevo ecocardiograma que demostró mejoría de la función ventricular (FEVI del $45 \%$ con hipocinesia leve anteroapical). Fue egresado con estatina, ácido acetilsalicílico, acenocumarina, metoprolol y enalapril, con un INR de 3.0 a los 8 días del evento coronario, con cita a la consulta externa en 6 semanas para definir la mejor estrategia de revascularización periférica.

\section{Discusión}

Presentamos el caso de un varón de 27 años de edad con antecedente de tromboembolia pulmonar, quien llevaba varios años asintomático bajo terapia anticoagulante, pero que presentó en esta ocasión un sindrome coronario agudo debido a una trombosis primaria de la descendente anterior. No se encontraron lesiones aterosclerosas en la angiografía ni en el ultrasonido intravascular, por lo que no se utilizaron stents ni balones en su tratamiento intervencionista. Después de un cuadro de falla cardiaca aguda evolucionó satisfactoriamente, por lo que egresó con ácido acetilsalicílico y cumarínico (INR de 3.0), teniendo pendiente el tratamiento de la obstrucción arterial periférica.

Aunque otras patologías, además de la enfermedad coronaria, se deben tomar en cuenta en un paciente con SAF que presenta dolor precordial, sobre todo si coexiste con lupus u otras enfermedades autoinmunes $^{1}$, la presencia de dolor y cambios en el electrocardiograma sugerentes de infarto obligan al clínico a tratarlo como cualquier otro sujeto con sospecha de un síndrome coronario agudo, independientemente del antecedente de tromboembolia pulmonar o del uso de anticoagulantes.

El infarto agudo del miocardio es raro como manifestación inicial del SAF. En un estudio correspondió solo al $2.8 \%$ de los casos incidentes, pero durante el seguimiento lo desarrollaron el $5.5 \%$ de los pacientes $^{4}$. Se estima que la trombosis coronaria se presenta en el $5 \%$ de las personas a quienes se les detectan $\mathrm{AFL}^{2}$, y también se ha observado una mayor prevalencia de AFL en pacientes que sufren síndromes coronarios agudos respecto a aquellos con angina estable crónica ${ }^{5}$. Solo la circulación arterial cerebral es afectada con mayor frecuencia que la coronaria en los pacientes con SAF (infartos cerebrales, ataques isquémicos transitorios y demencia de origen vascu$(a r)^{2}$. Se han descrito mecanismos atípicos de obstrucción coronaria ${ }^{6}$. En este paciente se encontraron alteraciones en dos territorios arteriales aunadas al antecedente de tromboembolia venosa, sin que hubiera ninguna alteración neurológica en el interrogatorio y la exploración dirigidos.

Así mismo, la enfermedad coronaria crónica es más común en los sujetos con SAF que en la población general. En un estudio se observaron cambios miocárdicos sugerentes de isquemia en el $29.6 \%$ de los pacientes con SAF (asintomáticos en cuanto a lo cardiaco), lo cual es comparable a la incidencia de enfermedad coronaria observada en en sujetos diabéticos o con nefropatía terminal 7 . En este caso no se identificaron lesiones coronarias mediante angiografía en ninguno de los vasos, y se corroboró la ausencia de ellas mediante ultrasonido intracoronario en los segmentos proximal y medio de la descendente anterior y en el tronco coronario izquierdo. La necrosis miocárdica detectada mediante resonancia magnética se asocia más con el tiempo de evolución del SAF, la presencia de livedo y la positividad de anticuerpos anti- 32 -glucoproteína que con los factores de riesgo cardiovascular tradicionales?.

Se ha relacionado a los anticuerpos anticardiolipina y su cofactor $\beta 2$-glucoproteína I con aterosclerosis incipiente ${ }^{5}$. Además de los efectos favorables conocidos de las estatinas en los sujetos con síndromes coronarios agudos, se ha descrito que la activación endotelial mediada por anti- $\beta 2$-glucoproteína puede ser inhibida por las estatinas ${ }^{5}$, por lo que incluso en ausencia de placas coronarias se decidió indicar atorvastatina a este paciente, a quien consideramos de alto riesgo para desarrollar eventos cardiovasculares a largo plazo, aun cuando no tiene ninguno de los factores de riesgo coronario clásicos.

En estos individuos, la anticoagulación de por vida es obligada. Se recomienda un INR de 3.0-3.5, una meta más alta que para otros estados de hipercoagulación ${ }^{2}$. Los eventos trombóticos pueden recurrir incluso con INR en rango terapéutico ${ }^{6,8}$.

El síndrome puede coexistir con alteraciones genéticas, como el factor $V$ de Leiden, $u$ otros estados protrombóticos ${ }^{9}$. Incluso se ha descrito resistencia al clopidogrel en estos enfermos ${ }^{10}$. Por lo tanto, si los episodios trombóticos recurren a pesar de una anticoagulación adecuada, deben sospecharse otras enfermedades. 
La enfermedad arterial periférica también ha sido descrita en el SAF y puede tener diversas presentaciones $^{11,12}$. En este caso se decidió diferir el tratamiento de esta alteración hasta que el paciente se recuperara del evento coronario. Aunque la morfología de la lesión favorecía la opción de tratamiento endovascular, la topografía orientaba más a una resolución quirúrgica.

\section{Conclusión}

Debido al gran espectro clínico del SAF, cualquier manifestación clínica en un paciente con este diagnóstico debe alertar al clínico sobre un posible evento trombótico arterial o venoso en prácticamente cualquier localización, o bien una reactivación de la enfermedad a través de otro mecanismo. En este caso, mediante la aspiración del trombo se logró resolver una oclusión trombótica total aguda de la descendente anterior sin necesidad de realizar angioplastia con balón o stent, ya que la trombosis no era asociada a una placa aterosclerótica.

\section{Bibliografía}

1. Miranda $\mathrm{CH}$, Gali LG, Marin-Neto JA, et al. Coronary thrombosis as the first complication of antiphospholipid syndrome. Arq Bras Cardiol. 2012;98:e66-9.

2. Asherson RA, Cervera R. Antiphospholipid antibodies and the heart; lessons and pitfalls for the cardiologist. Circulation. 1991;84:920-3.

3. Miyakis S, Lockshin MD, Atsumi T, et al. International consensus statement on an update of the classification criteria for definite antiphospholipid syndrome (APS). J Thromb Haemost. 2006;4:295-306.

4. Cervera R, Piette J, Font J, et al. Antiphospholipid syndrome: clinical and immunologic manifestations and patterns of disease expression in a cohort of 1,000 patients. Arthritis Rheum. 2002;46:1019-27.

5. Long BR, Leya $F$. The role of antiphospholipid syndrome in cardiovascular disease. Hematol Oncol Clin N Am. 2008;22:79-94.

6. Vianna JL, Khamashta M, Ordi-Ros J, et al. Comparison of the primary and secondary antiphospholipid syndrome: a European multicenter study of 114 patients. Am J Med. 1994;96:3-9.

7. Sacré K, Brihaye B, Hyafil F, et al. Asymptomatic myocardial ischemic disease in antiphospholipid syndrome. Arthritis Rheum. 2010;62:2093-100.

8. Monteiro AV, Branco LM, de Sousa L, et al. Antiphospholipid syndrome: a recurrent cardiac thromboembolic insult in spite of optimal anticoagulation. Reumatol Clin. 2015;11:177-8.

9. Santos JL, Cruz I, Herrero FM, et al. Trombosis coronaria recurrente, síndrome antifosfolipídico primario, factor $\mathrm{V}$ Leiden y virus de la inmunodeficiencia humana. Rev Esp Cardiol. 2004;57:997-9.

10. Middlebrooks $E H$, Panda $M$. Multiple recurrent stent thrombosis in a patient with coexisting clopidogrel resistance and increased anticardiolipin antibodies: a case report. Case Rep Med. 2010;2010:1-5.

11. Toffon A, Piovesan R, Minniti CF, et al. Lower limb ischemia: aortoiliac thrombosis related to antiphospholipid syndrome (APS) - case report and review of literature. Case Rep Surg. 2013;2013:536971.

12. Namazi MH, Kaheshi I, Serati AR, et al. Resistant hypertension due to unilateral renal artery occlusion as the first presentation of antiphospholipid syndrome. Cardiovasc Revasc Med. 2015;16:190-1. 